\title{
Combined Use of Mammography and FNA Eliminates Pitfalls in the Management of Metaplastic Breast Carcinoma
}

\author{
DIAMANTIS I. TSILIMIGRAS ${ }^{1}$, IOANNIS NTANASIS-STATHOPOULOS ${ }^{1}$, DIMITRIOS SCHIZAS ${ }^{2}$, \\ ANARGYROS BAKOPOULOS ${ }^{3}$, DEMETRIOS MORIS ${ }^{4}$, GABRIELA-MONICA STANC ${ }^{5}$, \\ ANASTASIOS TENTOLOURIS ${ }^{6}$, SOUMPHI NASSAR $^{7}$ and CHARITINI SALLA ${ }^{8}$ \\ ${ }^{1}$ School of Medicine, National and Kapodistrian University of Athens, Athens, Greece; \\ ${ }^{2}$ First Department of Surgery, and ${ }^{6}$ First Department of Propaedeutic and Internal Medicine, \\ Laikon General Hospital, School of Medicine, National and Kapodistrian University of Athens, Athens, Greece; \\ ${ }^{3}$ Third Department of Surgery, Attikon General Hospital, School of Medicine, \\ National and Kapodistrian University of Athens, Athens, Greece; \\ ${ }^{4}$ Division of Surgical Oncology, Department of Surgery, \\ The Ohio State University Wexner Medical Center, Columbus, OH, U.S.A.; \\ Departments of ${ }^{5}$ Pathology, and ${ }^{8}$ Cytopathology, Hygeia Hospital, Athens, Greece; \\ ${ }^{7}$ Department of Surgery, Kyanous Stavros Hospital, Athens, Greece
}

\begin{abstract}
Background: Due to its heterogeneity, metaplastic breast carcinoma (MBC) poses diagnostic dilemmas, leading to delayed treatment, thereby aggravating the prognosis. Over the years, there has been controversy regarding the role of fine-needle aspiration (FNA) cytology in timely diagnosis. Case Report: A 54-year-old woman presented with a palpable mass in the upper outer quadrant of her right breast with corresponding mammographic findings and FNA was performed. The smears revealed small-and medium-sized cohesive clusters of malignant cells with atypical nuclei. Sporadically, there was a pleomorphic population of notably large mononuclear cells, with disturbance of nuclear/cytoplasmic ratio, and binucleated or multinucleated malignant cells. The presence of chondromyxoid substance with focally embedded cells in a magenta-colored substrate was predominant in the background. These features, along with the corresponding mammographic findings, allowed for high preoperative suspicion of MBC. Surgical resection followed immediately without neoadjuvant chemotherapy; the pathology report led
\end{abstract}

This article is freely accessible online.

Correspondence to: Diamantis I. Tsilimigras, School of Medicine, National and Kapodistrian University of Athens, 75 Mikras Asias str., 11527, Athens, Greece. Tel: +30 2107716042, e-mail: tsilidiam@gmail.com

Key Words: Breast cancer, metaplastic carcinoma, fine-needle aspiration cytology, case report. to the definite diagnosis of $M B C$. Discussion: $M B C$ is a rare clinical entity with unfavorable prognosis, thus early diagnosis is imperative regarding its management. The effectiveness of FNA in the diagnostic algorithm has been questioned, with data from literature being rather contradictory. FNA seems to provide valuable information, which should always be interpreted in correlation with the clinical and mammographic findings. Conclusion: High preoperative suspicion of $M B C$ with the combination of mammography and FNA cytology necessitated the surgical excision of the lesion as the principal treatment approach. Although the role of FNA in the diagnosis of $M B C$ is debatable, its combination with clinical presentation and corresponding mammographic findings may prevent the administration of neoadjuvant chemotherapy in patients with ambiguous indications, given the poor response rate of this cancer subtype.

Metaplastic breast cancer (MBC) constitutes a rare group of primary mammary malignancies, with a prevalence of less than $1 \%$ of all breast lesions (1). It is an aggressive tumor that commonly presents as a palpable, rapidly growing breast mass in women who have usually entered their fifth decade of life (2). Over the years, there has been controversy concerning the effectiveness of fine-needle aspiration (FNA) cytology in the diagnosis of MBC (3-5), while many authors consider it a reliable option $(6,7)$. Herein, we present a case of a patient with MBC in whom breast digital mammography and FNA necessitated the excision of the lesion, which eventually turned out to be malignant. 


\section{Case Report}

A 54-year-old woman with a prior history of chronic lymphocytic lymphoma was referred for a mammogram and FNA cytological examination. On clinical examination, she presented with a palpable mass in the upper outer quadrant of her right breast and stated that the lesion had first appeared during self-examination 1 month earlier. She had a 30 packyear smoking history and, concerning her gynecological history, menarche occurred at 12 years, while menopause at 45 years of age. In comparison to a mammography report 4 years before, the new one revealed a high-density lesion with indistinct margins in accordance with the palpable finding (Figure 1). Therefore, further investigation was suggested.

FNA cytology revealed small- and medium-sized cohesive clusters of malignant cells with atypical nuclei. Sporadically, there was a pleomorphic population of notably large mononuclear cells, with disturbance of nuclear/cytoplasmic ratio, binucleated or multinucleated malignant cells. In the background, the presence of chondromyxoid substance in a magenta-colored substrate was predominant and a small number of cells, focally embedded in this substance, were also observed (Figure 2a).

The above mentioned cytological features along with the radiographic findings led to high suspicion of $\mathrm{MBC}$. The mass was subsequently resected and the pathological report revealed morphological and immunohistochemical characteristics (positivity for vimentin, CK14, CK5/6 and p63) of a matrix-producing metaplastic carcinoma in a chondromyxoid substrate, as well as multiple foci of necrosis (Figure 2b). According to the American Joint Committee on Cancer and the Union for International Cancer Control (AJCC/UICC) staging system, the surgical pathological report indicated a stage IIA (pT2N0) matrix-producing metaplastic carcinoma with a maximum diameter of $2.5 \mathrm{~cm}$. The tumor status was determined as estrogen receptor (ER)negative, progesterone receptor (PR)-negative and grade 2 human epidermal growth factor receptor 2 (HER2) positivity by immunohistochemistry. Subsequently, fluorescence in situ hybridization was used to confirm the amplification and HER2 positivity was eventually established. Additionally, right axillary lymph nodes were resected and no evidence of metastatic disease was present. Finally, the patient received adjuvant chemotherapy, including docetaxel, carboplatin and trastuzumab. No radiotherapy was performed due to sustained hematological toxicity. During the past 3 years, no sign of MBC relapse has manifested.

\section{Discussion}

As shown by a recent multicenter case-control study (8), MBC is characterized by larger size, more rapid growth, less frequent lymphovascular invasion, significantly lower positivity rates

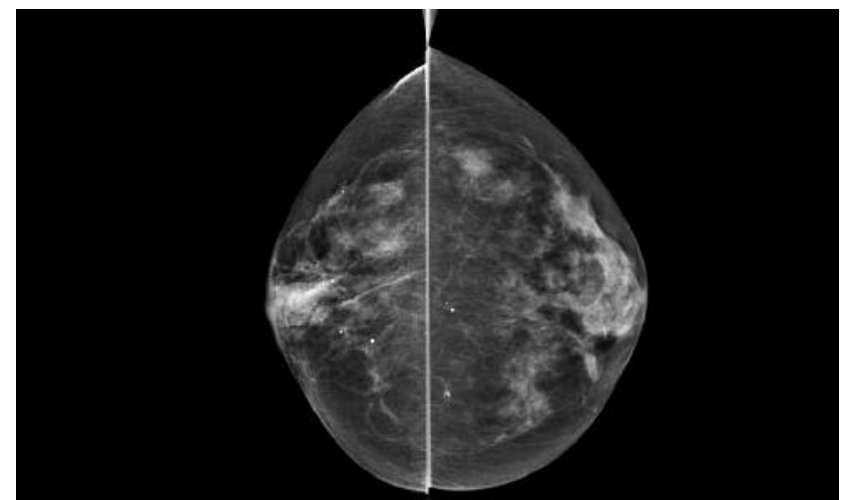

Figure 1. Digital mammography showing a high-density region with indistinct margins in the right breast.

for ER, PR and HER2, and more frequent triple-negative subtype, in contrast to invasive ductal carcinomas (IDC). Conversely, epidermal growth factor receptor (EGFR), p53, and cytokeratin 5/6 (CK5/6) are found at higher rates in patients with MBC. Furthermore, MBC usually presents with a lower incidence of lymph node involvement compared with IDC, but it displays a higher rate of distant metastasis, as well as higher tumor grade (9). All these clinicopathological features, along with the unfavorable prognosis related to this specific subtype of breast cancer, render timely diagnosis imperative regarding its management (3).

Due to its heterogeneity as a tumor, there are no known specific radiological features of MBC (10). All palpable masses should, therefore, be investigated with both mammography and ultrasound in order to increase the diagnostic accuracy, but most agree that definitive diagnosis is achieved by surgical excision (11).

Recent data from the Breast Cancer Surveillance Consortium have shown that the sensitivity and the specificity of digital mammography in breast cancer diagnosis lie at the level of $87.8 \%$ (95\% confidence interval $(\mathrm{CI})=87.3-88.4 \%)$ and $90.5 \%(95 \% \mathrm{CI}=90.4-90.6 \%)$, respectively (12). On the other hand, FNA is a method highly dependent on the skill and training of the aspirator and the interpreter. Thus, reported sensitivity ranges from $43.8 \%$ to $95 \%$, with specificity from $89.8 \%$ to $100 \%$ (13). Given the low possibility of occult disease in negative mammographies, it is essential that these two techniques be combined for all palpable breast lesions in order to minimize the risk of misdiagnosis.

Although many authors consider FNA a challenging and problematic procedure in diagnosing this specific subtype of breast cancer (3-5), its preoperative role should not be underestimated, as it seems to be a valuable diagnostic tool. According to the literature, cytological features of $\mathrm{MBC}$ are as follows: highly cellular smears; malignant cells in a 

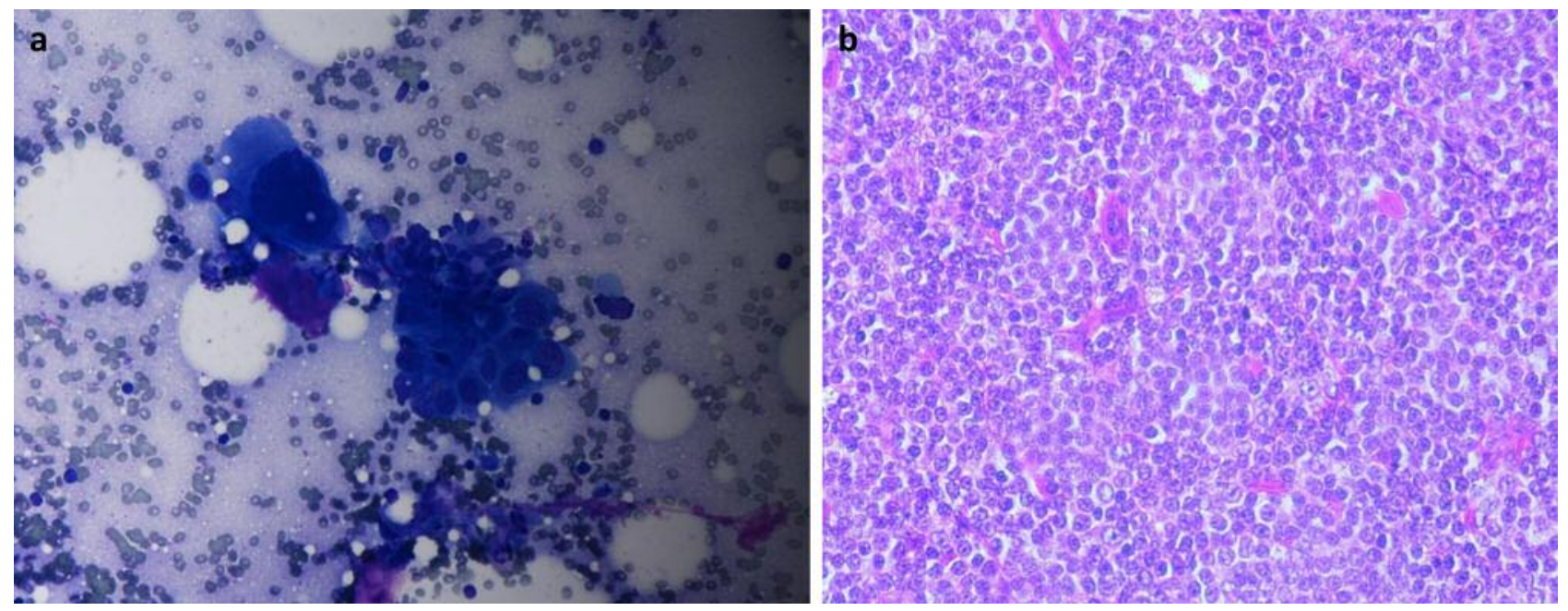

Figure 2. a: Fine-needle aspiration cytology leading to high suspicion of metaplastic breast carcinoma (MBC). b: Hematoxylin and eosin-stained section at $\times 40$ magnification showing characteristics of MBC.

myxoid background, which can be fibrillar or metachromatic; elongated, atypical and pleomorphic mesenchymal cells; multinucleated forms of malignant or benign nature; single or clustered carcinoma cells; and the presence of abnormal mitotic figures (14). In our case, only some of these features were revealed, therefore cytology was unable to establish an accurate diagnosis of MBC since both mesenchymal and squamous elements were not shown (14). However, FNA smear pointed out the mass as being positive for malignancy and, in combination with mammography findings, the diagnosis of MBC was highly suspected preoperatively. Consequently, the necessity for its surgical resection was established.

By definition, the term 'metaplastic' refers to differentiation of neoplastic epithelium into a non-epithelial phenotype comprising squamous cells and mesenchymal elements (1). Given the fact that $\mathrm{MBC}$ refers to a heterogenous group of neoplasms, as aforementioned, the cytological features are variable, and, therefore the cytological diagnosis is rendered fairly challenging. Nogueira et al. reported that some cytological findings, namely a liquid aspirate, a proteinaceous or chondromyxoid background, multinucleated giant cells from a poorly differentiated tumor and high nuclear pleomorphism, may suggest the diagnosis of MBC (15). Although these findings are relatively common in $\mathrm{MBC}$ cases, they are not specific and, for this reason, both carcinomatous and metaplastic components are required to be demonstrated in FNA smears in order for the diagnosis to be definite. Stanley et al. had earlier shown that FNA revealed both ductal carcinoma and metaplastic component only in four out of seven cases (57\%) and, thus, accurate diagnosis was not feasible in almost half of the cases (16).
Another study showed that smears containing solely malignant glandular elements on cytological examination ultimately revealed malignant squamous components on surgical resection specimens, thereby leading to the conclusion that the combination of cytological and histological examination should always be considered as it could eliminate pitfalls in diagnostic procedure (17). Finally, Kato et al. suggested that FNA cytology may permit MBC diagnosis but in order to increase its effectiveness, the tumor should be aspirated from different positions so that several cell components are adequately sampled (6).

Since MBC usually presents as stage III and IV disease with a higher incidence than IDC $(2,9)$, improving timely diagnosis and initiating the appropriate treatment is considered crucial for increasing life expectancy. As many studies have shown, the response of $\mathrm{MBC}$ to both neoadjuvant and adjuvant chemotherapy has been poor over the years $(2,5,9$, 18). In that context, most agree that surgery should be the first therapeutic approach when facing this type of cancer $(2,18)$. However, in cases of HER2-positive and triple-negative breast tumors, neoadjuvant chemotherapy may be considered if the appropriate criteria are met (19). High preoperative suspicion of MBC through combination of mammography and FNA cytology could prevent the administration of neoadjuvant chemotherapy in cases of ambiguous indications.

\section{Conclusion}

$\mathrm{MBC}$ is a rare clinical entity that usually poses diagnostic dilemmas because of its heterogeneity as a tumor. The response of $\mathrm{MBC}$ to neoadjuvant and adjuvant chemotherapy has been poor over the years. The combination of 
mammogram along with the FNA cytology allows for high preoperative suspicion of $\mathrm{MBC}$ and suggests surgical excision as the primary treatment approach while preventing the administration of ineffective neoadjuvant chemotherapy.

\section{References}

1 Abouharb S and Moulder S: Metaplastic breast cancer: clinical overview and molecular aberrations for potential targeted therapy. Curr Oncol Rep 17(3): 431, 2015.

2 Schwartz TL, Mogal H, Papageorgiou C, Veerapong J and Hsueh EC: Metaplastic breast cancer: histologic characteristics, prognostic factors and systemic treatment strategies. Exp Hematol Oncol 2(1): 31, 2013.

3 Luini A, Aguilar M, Gatti G, Fasani R, Botteri E, Brito JA, Maisonneuve P, Vento AR and Viale G: Metaplastic carcinoma of the breast, an unusual disease with worse prognosis: the experience of the European Institute of Oncology and review of the literature. Breast Cancer Res Treat 101(3): 349-353, 2007.

4 Lui PC, Tse GM, Tan PH, Jayaram G, Putti TC, Chaiwun B, Chan NH, Lau PP, Mak KL and Khin AT: Fine-needle aspiration cytology of metaplastic carcinoma of the breast. J Clin Pathol 60(5): 529-533, 2007.

5 Soler Monso MT, Catala I, Terricabras M, Petit A, Climent F, Perez-Casanovas L, Guma A and Morilla I: Metaplastic carcinoma of the breast with chondroid differentiation (matrixproducing carcinoma): study of the diagnostic cost-effectiveness of fine-needle aspiration biopsy and needle core biopsy. Acta Cytol 58(1): 9-14, 2014.

6 Kato T, Tohnosu N, Suwa T, Takahashi H, Tokuizumi M, Uehara $\mathrm{T}$ and Kobayashi TK: Metaplastic breast carcinoma with chondrosarcomatous differentiation: fine-needle aspiration cytology findings. A case report. Diagn Cytopathol 34(11): 772775, 2006.

7 Bagga PK, Mittal A and Mahajan NC: Matrix-producing carcinoma of the breast: fine-needle aspiration cytology findings of a rare subtype of metaplastic carcinoma. Cytopathology 22(6): 421-422, 2011.

8 Yu JI, Choi DH, Huh SJ, Ahn SJ, Lee JS, Shin KH, Kwon Y, Kim YB, Suh CO, Kim JH, Cho J, Kim IA, Lee JH and Park W: Unique characteristics and failure patterns of metaplastic breast cancer in contrast to invasive ductal carcinoma: a retrospective multicenter case-control study (KROG 13-07). Clin Breast Cancer 15(2): e105-115, 2015.
9 Lai HW, Tseng LM, Chang TW, Kuo YL, Hsieh CM, Chen ST, Kuo SJ, Su CC and Chen DR: The prognostic significance of metaplastic carcinoma of the breast (MCB) - a case-controlled comparison study with infiltrating ductal carcinoma. Breast 22(5): 968-973, 2013.

10 Castella E, Gomez-Plaza MC, Urban A and Llatjos M: Fineneedle aspiration biopsy of metaplastic carcinoma of the breast: report of a case with abundant myxoid ground substance. Diagn Cytopathol 14(4): 325-327, 1996.

11 Zhang Y, Lv F, Yang Y, Qian X, Lang R, Fan Y, Liu F, Li Y, Li $\mathrm{S}$, Shen B, Pringle GA, Zhang $\mathrm{X}, \mathrm{Fu} \mathrm{L}$ and Guo $\mathrm{X}$ : Clinicopathological features and prognosis of metaplastic breast carcinoma: experience of a major Chinese Cancer Center. PLoS One 10(6): e0131409, 2015.

12 Sprague BL, Arao RF, Miglioretti DL, Henderson LM, Buist DS, Onega T, Rauscher GH, Lee JM, Tosteson AN, Kerlikowske K, Lehman CD and Breast Cancer Surveillance C: National Performance Benchmarks for Modern Diagnostic Digital Mammography: Update from the Breast Cancer Surveillance Consortium. Radiology 283(1): 59-69, 2017.

13 Simsir A, Rapkiewicz A and Cangiarella J: Current utilization of breast FNA in a cytology practice. Diagn Cytopathol 37(2): 140142, 2009.

14 Gatuso P, Reddy VB and Masood S: Differential Diagnosis in Cytopathology. Cambridge University Press, 2010.

15 Nogueira M, Andre S and Mendonca E: Metaplastic carcinomas of the breast - fine needle aspiration (FNA) cytology findings. Cytopathology 9(5): 291-300, 1998.

16 Stanley MW, Tani EM and Skoog L: Metaplastic carcinoma of the breast: fine-needle aspiration cytology of seven cases. Diagn Cytopathol 5(1): 22-28, 1989.

17 Lale S, Kure K and Lingamfelter D: Challenges to diagnose metaplastic carcinoma of the breast through cytologic methods: an eight-case series. Diagn Pathol 6: 7, 2011.

18 Tzanninis IG, Kotteas EA, Ntanasis-Stathopoulos I, Kontogianni $\mathrm{P}$ and Fotopoulos G: Management and outcomes in metaplastic breast cancer. Clin Breast Cancer 16(6): 437-443, 2016.

19 Loibl S, Denkert C and von Minckwitz G: Neoadjuvant treatment of breast cancer--Clinical and research perspective. Breast 24(Suppl 2): S73-77, 2015.

Received April 1, 2017

Revised April 14, 2017

Accepted April 19, 2017 